\title{
Regulating the regulator of ROS production
}

\author{
Cell Research (2014) 24:908-909. doi:10.1038/cr.2014.66; published online 20 May 2014
}

\begin{abstract}
Balancing inflammatory reactive oxygen species (ROS) production is essential for safely eliminating pathogenic microbes. The newly described protein Negative Regulator of ROS (NRROS) dampens ROS production by restricting NOX2 availability, and thus "cools-off" inflammation.
\end{abstract}

Oxygen-based metabolism was essential for proliferation and diversification of life on Earth but imposed the need to cope with reactive oxygen species (ROS) as an unavoidable aspect of aerobic respiration. Multicellular organisms have evolved a host of mechanisms to utilize ROS to their advantage, thus employing them as mediators of cellular signaling and bactericidal agents against invading pathogens. Excessive ROS production, however, results in oxidative tissue injury, and as recently shown, may participate in the etiology of autoimmunity [1]. Collateral oxidative cell damage is inseparable from the host innate immunologic responses, underscoring the necessity of regulatory mechanisms that modulate ROS production. A recent publication in $\mathrm{Na}$ ture has identified a crucial mechanism that balances ROS production and thus modulates the inflammatory response [1]. Noubade et al. [1] describe a protein Negative Regulator of ROS (NRROS, a.k.a. Lrrc33) that interacts with NOX2 (gp91phox) and affects its stability. The NRROS-bound NOX2 in the endoplasmic reticulum (ER) was targeted for proteosomal degradation, and thereby NRROS limited NOX2-derived ROS by impeding NOX2 trafficking to the phagosomal membrane. The authors showed that NRROS and p22phox competed for nascent gp91phox in the ER. Binding to $\mathrm{p} 22$ phox stabilized gp91phox allowing subsequential trafficking and assembly of the functional NOX2 at the plasma membrane whereas binding to NRROS induced gp91phox trafficking to ER-associated protein degradation (ERAD) pathway (schematically summarized in Figure 1).

NOX2 is a major source of superoxide radicals produced by phagocytic cells during the oxidative burst. Thus, an important discovery made is that NRROS is downregulated in response to priming by pro-inflammatory stimuli including lipopolysaccharide and type II interferon. NRROS deficiency resulting from exposure to these stimuli caused the cells to respond to subsequent inflammatory challenges with an appropriately robust ROS burst required for killing bacteria and viruses. The kinetics of ROS production in stimulated NRROS-deficient versus wild-type cells was identical, suggesting that NRROS functions to establish the upper limit in the achievable levels of ROS production following phagocyte activation. These observations provide important insights into the widely recognized but poorly understood amplifying effect of basal inflammation on the subsequent activation of innate immune responses. The implication is that chronic exposure to low levels of pro-inflammatory mediators can exacerbate the availability of functional NOX2 by chronically repressing NRROS. In support of this idea, NRROS-knockout mice were better equipped to fight infection by L. monocytogenes and survived better in contrast to wild-type animals, consistent with the known effect of ROS in promoting pathogen killing. On the flipside, however, elimination of a central repressive mechanism limiting NOX2-derived ROS induced lethal encephalomyelitis (EAE) with rapid deterioration of the central nervous system in immunized NRROS-knockout mice [1]. A possible clinically relevant interpretation of this observation is that while severe oxidative stress facilitates elimination of pathogenic organisms, it can also overwhelm the host's ability to clear oxidized biomolecules which may trigger the onset of autoimmunity. The findings raise the intriguing question whether dysregulated ROS production during successive acute inflammatory events is conducive to the pathogenesis of autoimmune disorders. This concept also resonates with some earlier studies highlighting the effects of environmentinduced chronic systemic inflammation and the propensity of mammals to develop degenerative neurological disorders [2, 3]. For example, it was observed that exacerbated NOX2 activity underlies microglia-mediated neurotoxicity that can lead to Parkinson's and Alzheimer's diseases [2]. It was previously theorized that chronic low-grade inflammatory states promoted by exposure to environmental toxicants (air pollution, pesticides, etc.) primes microglia (the macrophages of the brain) to produce exaggerated amounts of ROS generated largely by NOX2 [3]. As demonstrated by studies testing this hypothesis, microglia from animals exposed to diesel exhaust particles produced robust bursts of ROS when subsequently challenged with lipopolysaccharides [2]. Levels of ROS produced by primed microglia were demonstrated to be neurotoxic to dopaminergic neurons and induce 


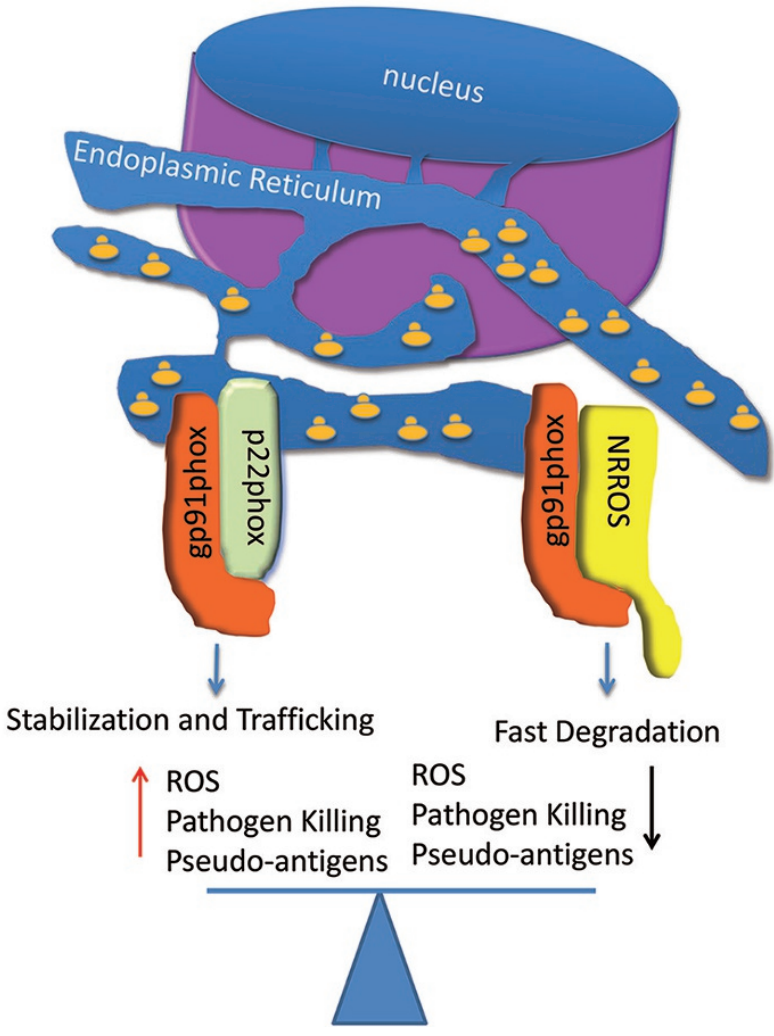

Figure 1 Nascent NOX2 interactions in the ER. NOX2 is a major source of superoxide radical anion during phagocytic cell activation. Interaction of nascent NOX2 with p22phox stabilizes NOX2 in the ER and allows for the maturation and trafficking of NOX2 to the plasma membrane where it becomes functional. NRROS is a newly identified NOX2-binding partner in the ER. Binding of NOX2 by NRROS accelerates NOX2 degradation, and thereby modulates ROS production by reducing NOX2 level.

neurodegeneration in a mouse model [4]. Other studies showed that feedback signaling by NOX2-derived ROS limits $\mathrm{TNF} \alpha$ and interleukin- 6 expression by activated macrophages, which alleviated acute inflammatory lung injury [5]. The production of ROS by specialized systems is currently considered to be limited by cofactor and $\mathrm{O}_{2}$ availability [6]. The identification of NRROS and its function indicates that on the contrary specific mechanisms exist to dynamically regulate the levels of ROS. Taken together, these studies suggest that budgeting ROS production is indispensable for safe interactions of the host with the environment. They also suggest that exacerbated ROS production leads to production of pseudo-antigens and oxidized biomolecules whose clear- ance may be the rate-limiting factor, and hence the necessity to limit their production by dampening ROS generation. This provocative hypothesis is consistent with the finding of higher levels of malonaldehyde (MDA)-adduct proteins after immunization in NRROSdeficient mice [1]. Another bit of evidence supporting this idea is the finding that administration of ROS scavengers after the onset of autoimmune EAE reduced the lethality of immunized NRROS-knockout mice back to wildtype control levels [1]. Although the levels of oxidized proteins were not assessed after ROS scavenger treatment, the near abrogation of autoimmune EAE by ROS scavengers administered after the onset of EAE indicates that resolution of ongoing oxidative stress (or the clearance of oxidized pseudoantigens) is sufficient to prevent further neurologic deterioration.

The role of ROS as mediators of tissue damage has been established (see reviews $[7,8])$. To date, much less is understood about the role of ROS in regulating the initiation, intensity, localization, and resolution of the inflammatory process. As the redox sensitivity of different signaling cascades varies, the concentrations and kinetics of ROS production are likely to shape specific inflammatory programs. Dysregulated ROS generation as shown by this study [1] is likely to cause detrimental effects produced by inflammation gone awry. Addressing questions related to the integration of redox signaling in inflammation and temporal control of differential ROS fluxes will further our understanding about the role of ROS in modulating inflammation and will provide exciting opportunities for therapeutic interventions.

\section{Marcelo G Bonini1 ${ }^{1,2,3}$, Asrar B Malik ${ }^{1,4}$}

${ }^{1}$ Department of Pharmacology, ${ }^{2}$ Department of Medicine, ${ }^{3}$ Department of Pathology, ${ }^{4}$ Center for Lung and Vascular Biology, The University of Illinois College of Medicine, 835 South Wolcott Avenue, Chicago, IL 60612, USA

Correspondence: Asrar B Malik

E-mail:abmalik@uic.edu

\section{References}

1 Noubade R, Wong K, Ota N, et al. Nature 2014; 509:235-239.

2 Levesque S, Taetzsch T, Lull ME, et al. $J$ Neurochem 2013; 125:756-765.

3 Block ML, Zecca L, Hong JS. Nat Rev Neurosci 2007; 8:57-69.

4 Qin L, Wu X, Block ML, et al. Glia 2007; 55:453-462.

5 Di A, Gao XP, Qian F, et al. Nat Immunol 2012; 13:29-34.

6 Gupte SA, Levine RJ, Gupte RS, et al. J Mol Cell Cardiol 2006; 41:340-349.

7 Rader DJ, Ischiropoulos H. Nat Med 2007; 13:1146-1147.

8 Koenitzer JR, Freeman BA. Ann N Y Acad Sci 2010; 1203:45-52. 